\title{
Apoptosis-resistant T cells in SLE: role for the regulatory B subunits of PP2A phosphatase
}

"T cells from patients with SLE are known to be resistant to apoptosis after cell activation," states George Tsokos, from the Beth Israel Deaconess Medical Center at Harvard Medical School, but the mechanisms behind this abnormality are not well understood. A paper published in the Proceedings of the National Academy of Sciences shows that altered activity of the serine/threonine phosphatase PP2A could be responsible, at least in some patients.

\section{4 ...inhibition of $B \beta$... protected T cells from apoptosis induced by withdrawal of IL-2... 77}

PP2A is a ubiquitously expressed enzyme comprising three subunits: a scaffold protein (A), a catalytic subunit (C) and a regulatory subunit (B). As their name suggests, the regulatory $B$ subunits control the enzyme's activity; these subunits have a tissue-specific expression pattern. Previous work from
Tsokos' group showed that the activity and expression of the A subunit of PP2A is increased in patients with SLE; in this study, they turn their attention to the B subunits.

"First, we determined that a particular B subunit-B $\beta$ - was present at low levels in unstimulated $\mathrm{T}$ cells, disappeared after cell activation and was induced by low levels of IL-2," explains Tsokos. "Next, we cloned the gene for $\mathrm{B} \beta$ in order to study its function." Overexpression of $\mathrm{B} \beta$ in vitro in primary $\mathrm{T}$ cells increased levels of apoptosis compared with vector alone. In addition, inhibition of $\mathrm{B} \beta$ using small interfering RNA protected T cells from apoptosis induced by withdrawal of IL-2.

On the basis of these findings, the authors decided to look at $\mathrm{T}$ cells from patients with SLE, to see if regulation of $\mathrm{B} \beta$ had a role in apoptosis in this disease setting. $\mathrm{T}$ cells from $\sim 50 \%$ of patients with SLE were protected from apoptosis induced by IL-2 withdrawal ('apoptosis-resistant' patients). T cells from the remaining patients had similar levels of apoptosis as the healthy controls ('apoptosis-susceptible' patients). The authors then assessed levels of $B \beta$ in the two groups of patients with SLE: $B \beta$ levels in $\mathrm{T}$ cells from the apoptosis-susceptible and normal patients increased $\sim$ threefold after IL-2 withdrawal, but levels of B $\beta$ in the apoptosis-resistant patients remained unchanged despite IL-2 withdrawal. "This suggests that altered function of $B \beta$ may be one of the causes of apoptosis resistance in T cells from SLE patients," says Tsokos. "It is important to determine why some patients fail to upregulate $\mathrm{B} \beta$ because this phenomenon could contribute to their disease."

Jenny Buckland

Original article Crispín, J. C. et al. Induction of PP2A $\beta$, a regulator of IL-2 deprivation-induced T-cell apoptosis, is deficient in systemic lupus erythematosus. Proc. Natl Acad. Sci. USA 108, 12443-12448 (2011) 